\title{
A Tale of Two Women: Is There a Benefit to Bilevel Ventilation in Pregnancy?
}

Laura Immordino, $M D$, and Adam Kaufman, $M D$

\section{Case Presentation 1}

The patient is a 24 year-old pregnant female at 31 and 6/7 weeks estimated gestational age, who presented to the emergency department with complaint of intermittent right-sided abdominal pain, nausea, vomiting and fevers over the past twenty-four hours. The patient's vital signs at presentation were significant for a heart rate of 130 beats/minute and a blood pressure of $90 / 32$ $\mathrm{mm} \mathrm{Hg}$. Her physical examination was otherwise unremarkable, and she was initially admitted to the maternal-fetal medicine service and started on intravenous fluids. Over the next 12 hours, the patient remained tachycardic with blood pressures in the $80 \mathrm{~s} / 40 \mathrm{~s}$. She began to develop respiratory distress, and an ICU evaluation was requested.

At the time of evaluation, the patient's physical exam revealed a well-developed, gravid woman in moderate distress. Her temperature was $100.7^{\circ}$ Fahrenheit, pulse was 136 beats/minute, blood pressure was $94 / 41 \mathrm{~mm} \mathrm{Hg}$, respiratory rate was 38 breaths/ minute, and pulse oximetry was $99 \%$ on a $100 \%$ non-rebreather mask. She was anicteric with moist mucous membranes and normal skin turgor. On cardiac examination, her heart rate was tachycardic and regular, without any murmurs. Her lungs were clear to auscultation and abdomen was soft and gravid without any tenderness to palpation. She had no peripheral edema. On vaginal exam, her cervix was long and one centimeter dilated.

Laboratory results were significant for a white blood cell (WBC) count of $19.8 \mathrm{~B} / \mathrm{L}$ and $\mathrm{CO} 2$ of $17 \mathrm{mmol} / \mathrm{L}$. Arterial blood gas was $7.46 / 25 / 125 / 18 / 99 \%$ on a $100 \%$ non-rebreather mask. Broad spectrum antibiotics were started, and a stat CT of the chest, abdomen and pelvis was performed, which showed dense consolidation of the left lower lobe, consistent with pneumonia.

The patient was maintained on a non-rebreather with stable oxygen saturations for several hours, but eventually decompensated, requiring emergent intubation. She was initially placed on assist-control ventilation; however, her oxygen saturations remained in the 60 s to 70 s. She was noted to have decreased left-sided breath sounds, despite pulling back the endotracheal tube. A stat chest radiograph was performed, which showed near complete collapse of the left lung.

The patient was subsequently switched to bilevel ventilation with eventual improvement in her oxygenation (see Figures 1 and 2). She initially required high level settings, with an inspiratory/ expiratory pressure of 35/10 $\mathrm{mm} \mathrm{Hg}$. The patient's status improved over the next few days with antibiotic treatment, and cultures all remained negative, but serologies were positive for Mycoplasma IgM. Pressure support was discontinued on hospital day four, and ventilatory support was titrated down slowly over the next few days. On hospital day seven, the patient was successfully extubated. Antibiotics were narrowed to oral

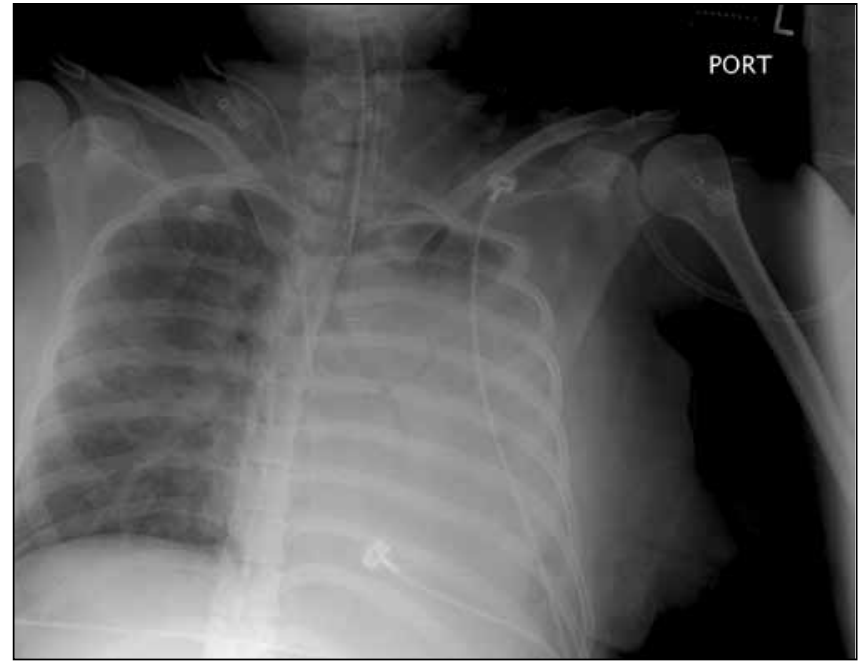

Figure 1. CXR following intubation on assist-control ventilation.

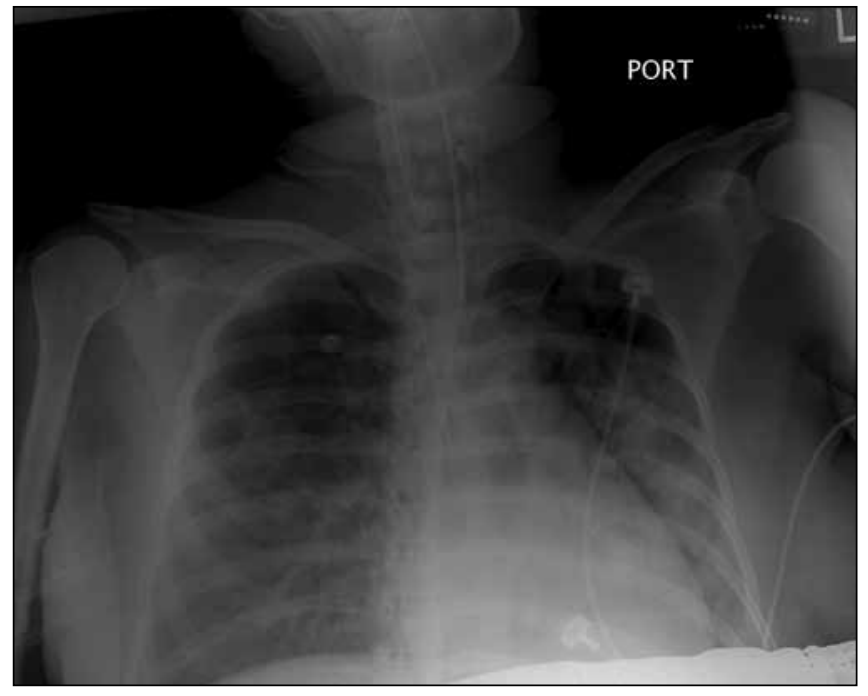

Figure 2. CXR following change to bi-level ventilation.

azithromycin to complete a fourteen day course of antibiotics for mycoplasma pneumonia.

\section{Case Presentation 2}

The patient is a 19 year-old pregnant woman at 29 and 6/7 weeks estimated gestational age, transferred from an outside hospital to the maternal-fetal medicine service with complaints of nausea, vomiting and fever for one week. Over the past twenty-four hours, the patient complained of increasing abdominal pain and developed fevers to $103^{\circ}$ Fahrenheit. 
The patient had no significant past medical or gynecologic history. She denied any previous surgeries or allergies to medications. On transfer from the outside hospital, she was receiving intravenous ampicillin, ceftriaxone, betamethasone and fluids.

Several hours after admission, the patient began to develop respiratory distress, and an ICU evaluation was requested. At that time, physical examination revealed a well-developed, gravid woman in moderate to severe distress. Her temperature was $102.3^{\circ}$ Fahrenheit, pulse was 127 beats/minute, blood pressure was $115 / 58 \mathrm{~mm} \mathrm{Hg}$, respiratory rate was 34 breaths/ minute, and pulse ox was $98 \%$ on a $100 \%$ non-rebreather. She was noted to have moist mucous membranes, scleral icterus, and tachycardia without murmurs. The remainer of the exam was significant for bilateral rales and mild abdominal tenderness to palpation diffusely, without rebound or guarding. She was noted to have mild lower extremity pitting edema. Neurologic exam was within normal limits.

Laboratory studies revealed a WBC count of 9.7 B/L, hemoglobin of $8.1 \mathrm{~g} / \mathrm{dL}$, and platelet count of $162 \mathrm{~B} / \mathrm{L}$. Her basic metabolic panel was within normal limits; however, liver panel showed elevated total and direct bilirubin levels of 4.8 and $2.2 \mathrm{mg} / \mathrm{dL}$, AST of $698 \mathrm{U} / \mathrm{L}$, ALT of $293 \mathrm{U} / \mathrm{L}$, and alkaline phosphatase of $193 \mathrm{U} / \mathrm{L}$. LDH was 1169 , fibrinogen was 326 , and D-dimer was elevated at 10.21. A diagnostic amniocentesis was performed, which showed no evidence of chorioamnionitis. Chest radiograph at that time showed bilateral infiltrates.

Hepatitis serologies, autoimmune, and vasculities studies were negative. Blood, urine, and bronchoalveolar lavage cultures for viral, bacterial, and fungal infections were negative. Hematologic work-up revealed no evidence of microangiopathic anemia, and HUS/TTP was ruled out. Echocardiogram showed no evidence of tamponade, lower extremity ultrasounds were negative for deep venous thromboembolism, and abdominal ultrasound showed no obvious liver pathology. Results from the outside hospital were only significant for a positive Mycoplasma IgM.

The patient remained febrile in the range of $102^{\circ}$ to $103^{\circ}$ Fahrenheit for nearly two weeks, despite empiric treatment with broad-spectrum antibiotics. On hospital day five, the patient's respiratory status deteriorated requiring intubation. She was placed on assist control ventilation with a respiratory rate of 20 , tidal volume of $400 \mathrm{cc}$, PEEP of 5, and $\mathrm{FiO} 2$ of $50 \%$. On hospital day seven, the patient's oxygenation dropped, requiring $100 \%$ FiO2. At this time, her ventilator settings were changed to ARDS protocol, and the FiO2 was titrated down over the next few days. On hospital day 16 , the patient was noted to have high peak inspiratory and plateau pressures, with $\mathrm{PaO} 2$ ranging from 65 to 90 . On hospital day 18, in order to facilitate weaning, the patient was placed on bilevel ventilation with inspiratory/ expiratory pressures of 30/5. Prior to the ventilatory switch, her $\mathrm{PaO} 2$ was 90 on $50 \% \mathrm{FiO} 2$; following the mode change, her $\mathrm{PaO} 2$ was 167 on $50 \% \mathrm{FiO} 2$. The inspiratory pressures were titrated down over the next few days, and on hospital day 23 she was placed on pressure support of 18 . She was weaned to a $40 \%$ tracheal collar on hospital day 27 , and transferred out of the ICU on hospital day 24 .

\section{Discussion}

Bilevel ventilation is a form of partial ventilatory support, which allows for spontaneous breathing at any point in the ventilator cycle. It is a time-triggered, pressure-limited,and time-cycled mode of ventilation. Inspiratory and expiratory pressures are set as PEEP high and PEEP low, and the physician is able to set length of time for the PEEP low. The goal of PEEP high is to help open the consolidated portions of the lungs that are often present in patients with acute lung injury or ARDS. PEEP low is a pressure release phase that allows for lung recoil and CO2 removal. A built-in valve allows for spontaneous breathing throughout the respiratory cycle. ${ }^{1,2}$ Spontaneous breathing has been shown to preferentially recruit dependent lung regions while simultaneously allowing for less neuromuscular blockade and sedation. ${ }^{3,4}$

In traditional mechanical ventilation, the majority of ventilation occurs centrally as opposed to at the distal alveoli, where the majority of perfusion occurs. The goal of bilevel ventilation is to increase alveolar recruitment which leads to decreased shunting, better arterial oxygenation, and improved ventilation-perfusion matching. ${ }^{7,8}$ By allowing for spontaneous breathing throughout the respiratory cycle, bilevel ventilation results in increased patient comfort and thus a decreased need for sedation and neuromuscular blockade. ${ }^{6}$ Since excessive sedation has been associated with a longer duration of intubation, decreased cough reflex, and increased risk of ventilator associated pneumonia, many studies have suggested that the use of bilevel ventilation can lead to a shorter ICU and hospital length of stay.

Contraindications to bilevel ventilation include patients with COPD (as they are at risk of barotrauma) and patients with neurologic disorders with increased intracranial pressure (as permissive hypercapnea is contraindicated in these patients).$^{1,6,8}$

\section{References}

1. Frawley PM, Habashi N. Airway Pressure Release Ventilation: Theory and Practice. AACN Clinical Issues 2001; 12(2): 234-46.

2. Habashi N. Other approaches to open-lung ventilation: Airway pressure release ventilation. Crit Care Med 2005; 33(3): S228-40.

3. Hering R, Peters D, Zinserling J et al. Effects of spontaneous breathing during airway pressure release ventilation on renal perfusion and function in patients with acute lung injury. Intensive Care Med 2002; 28: 1426-33.

4. Hering R, Viehofer ventilation on intestinal blood flow in experimental lung injury. Anesthesiology 2003; 99: 1137-44. A, Zinserling J et al. Effects of spontaneous breathing during airway pressure release

5. Hering R, Zinserling J, Wrigge $\mathrm{H}$ et al. Effects of Spontaneous Breathing During Airway Pressure Release Ventilation on Respiratory Work and Muscle Blood Flow in Experimental Lung Injury. Chest 2005; 128(4): 2991-8.

6. Putensen $\mathrm{C}$, Wrigge $\mathrm{H}$. Clinical Review: Biphasic positive airway pressure and airway pressure release ventilation. Critical Care 2004; 8(6): 492-7. 
7. Putensen C, Mutz N, Putensen-Himmer G et al. Spontaneous breting during ventilatory support improves ventilation-perfusion distributions in patients with acute respiratory distress syndrome. Crit Care Med 1999; 159: 1241-48.

8. Varpula T, Valta P, Niemi R et al. Airway Pressure Release Ventilation as a Primary Ventilatory mode in acute respiratory distress syndrome. ACTA Anaesthiol Scan 2004; 48: 722-31.

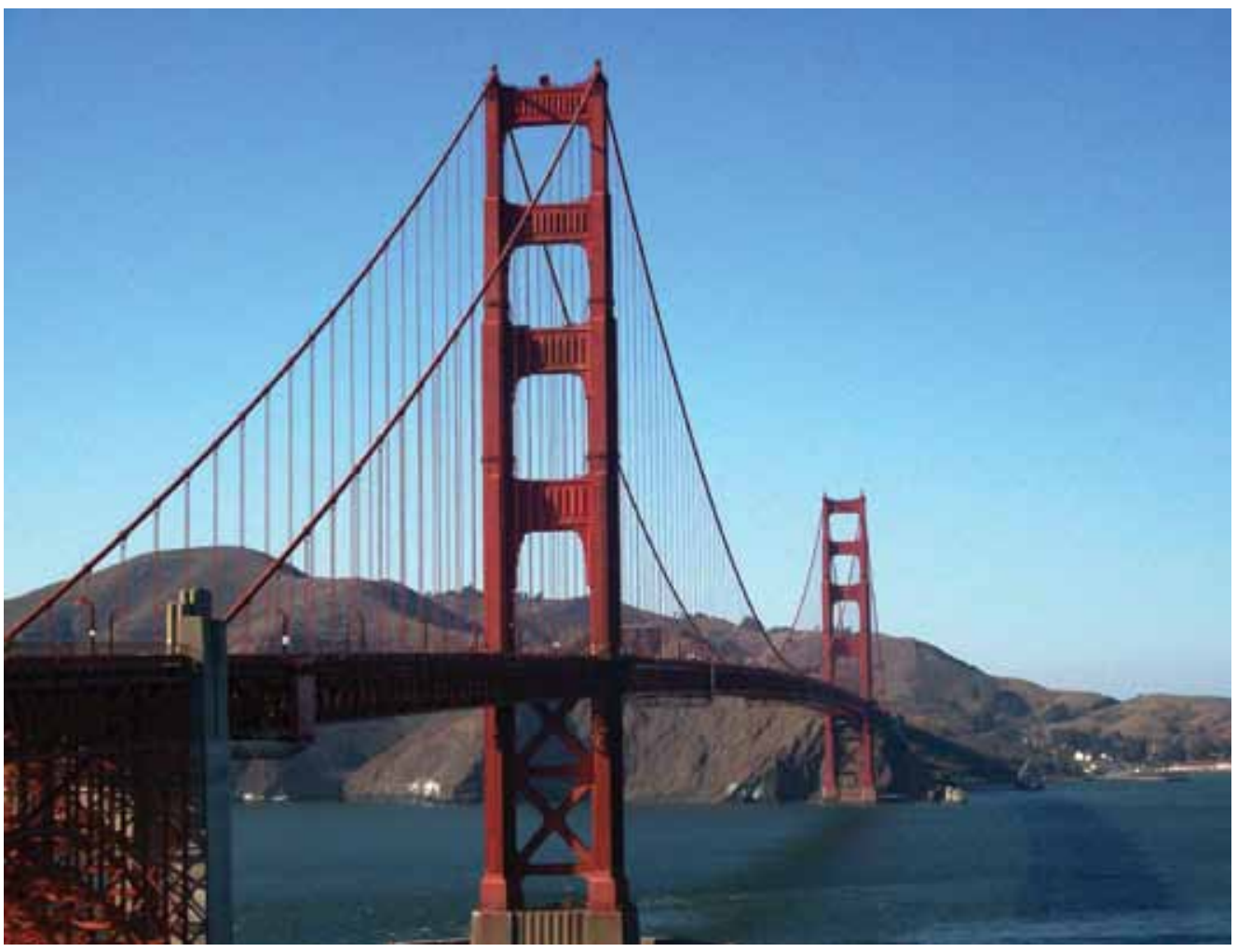

